\title{
Short-Term Intra Ocular Pressure Using Intravitreal
}

\section{Bevacizumab}

Yolanda Chávez-Romero ${ }^{1,2}$, Alba Delia Campaña-Salcido ${ }^{2,3,4}$, Efraín Romo-García ${ }^{1,2,5}$, José Alfredo Contreras-Gutiérrez ${ }^{3,4}$ and Anthon Álvarez-Arredondo ${ }^{2,3,4}$

1. Ophthalmology Department, Civil Hospital of Culiacán (HCC), Culiacán 80030, México

2. Center of Research and Teaching in Health Sciences (CIDOCS), Culiacán 80030, México

3. Faculty of Medicine, Autonomous University of Sinaloa, Culiacán 80019, México

4. Academic Group UAS CA-285, Culiacán 80030, México

5. High Specialty in Retina and Vitreous, Civil Hospital of Culiacán (HCC), Culiacán 80030, México

\begin{abstract}
At Ophthalmology Department of Civil Hospital of Culiacán, we investigated the inmediate effect of intravitreal injection of Bevacizumab on the IOP (intra ocular pressure) $30 \mathrm{~min}$ before and $24 \mathrm{~h}$ was measured after application of $0.1 \mathrm{~mL}$ of Bevacizumab (Avastin) to 67 patients under sterile technique in operating room, with topical anesthetic tetracaine at $3.5 \mathrm{~mm}$ from the sclerocorneal junction. Pre-application average IOP was $13.5 \pm 2.6 \mathrm{mmHg}$, and post-implementation IOP was $13.8 \pm 2.6 \mathrm{mmHg}$. Diagnostics: PDR + MESC (proliferative diabetic retinopathy with macular edema significant clinically) in 26 patients (38.8\%), NPDRSC (nonproliferative diabetic retinopathy significant clinical) in 14 patients (20.9\%), wet AMD (age related macular degeneration) in 14 patients (20.9\%), OCRV (occlusion central retinal vein) in 3 patients $(4.5 \%)$, OBRV (occlusion of branch retinal vein) in 3 patients (4.5\%), SCChR (serous central chorioretinopathy) in 2 patients (4.5\%), ChNnoA-R (choroidal neovascularization no age related) in 2 patients (3\%), CME (cystoid macular edema) in 2 patients $(3 \%)$.
\end{abstract}

Key words: Bevacizumab, intra ocular pressure, anti-VEGF, PDR, macular edema.

\section{Introduction}

Angiogenesis is a process that is essential for various physiological processes, such as embryonic and postnatal growth, reproductive functions and the scarring of wounds [1]. Multiple studies in recent decades have established that this process requires a number of steps in endothelial cells and murals where numerous families of ligands involved. However, despite the complexity, VEGF (vascular endothelial growth factor) appears to be necessary for the growth of blood vessels in normal or pathological circumstances [2].

\subsection{Antiangiogenic Therapy}

The abnormal growth of blood vessels, called

Corresponding author: Anthon Álvarez Arredondo, Ph.D., professor, research fields: pharmacology and toxicology. neovascularization, is a central feature of retinal disorders including retinopathy of prematurity, RVO (retinal venous occlusions), proliferative diabetic retinopathy and MDNEA-R (macular degeneration neovascular related to age), which are the leading causes of blindness in both childhood and productive adult life [3]. At present, it is known that inhibiting the angiogenic cascade and vascular permeability effective treatment in ocular neovascularization is achieved as in these pathologies [4]. It is noteworthy that, systemically, it is known that VEGF mRNA (messenger ribonucleic acid) is expressed in a wide variety of human tumors [5]. Then, it is known that this therapy is used successfully to treat different types of cancers such as colon cancer, gastrointestinal, kidney, among others [2].

The introduction of VEGF inhibitors (anti-VEGF) in ophthalmology, represented progress in treating 
many retinal diseases, once deemed untreatable. Bevacizumab (Avastin) is a murine humanized monoclonal antibody that is directed against all biologically active isoforms of VEGF, there are many studies reporting complications of intravitreal antiangiogenic. One of the reported adverse effects is largely increased intra ocular pressure due to the intravitreal injection.

\subsection{Applications in Ophthalmology}

\subsubsection{Retinopathy of Prematurity}

This disease is the number one blind children in Latin America cause. The first major study comparing conventional laser treatment with intravitreal Bevacizumab was the BEAT-ROP, in which it was found that monotherapy with Bevacizumab $(0.625 \mathrm{mg})$ compared with the laser, in infants with Stage 3+, showed benefits for the Area I, not for Zone II. It also noted that the peripheral retinal vessels continued to grow after treatment, contrary to laser treatment leading to a permanent destruction of the peripheral retina [6].

\subsubsection{MDNA-R}

The effectiveness of anti-VEGF (Pegaptanib, Ranibizumab and Bevacizumab) agents in terms of stability and improvement in visual acuity after 1 2 years of treatment is known. Ranibizumab and Bevacizumab have resulted in a comparable improvement together also beneficial visual effects are consistent with the effects on structural changes in the lesion size, in studies fluorangiography and optical coherence tomography [7]. Bevacizumab (Avastin) is a humanized murine monoclonal antibody produced by DNA (deoxyribonucleic acid) technology from Chinese hamster ovary cells. This antibody is directed against all biologically active isoforms of VEGF, has two binding sites for this factor and to join blocks the effects of VEGF and acts as an antiangiogenic. FDA (Food and Drugs Administration) approved Bevacizumab for the treatment of colorectal cancer, because of its low cost compared to other antiangiogenic, it has been used as an "off-label" since 2004 to treat some retinal diseases [8].

\subsubsection{Proliferative Diabetes Retinopathy}

There is currently a dose or treatment regimen specific so far [9]. They suggest that anti-VEGF can reduce the risk of intraocular hemorrhage in patients with PDR (proliferative diabetic retinopathy) [10].

\section{Materials and Methods}

Under informed consent, measurements of intraocular pressure in patients undergoing intravitreal Bevacizumab, $30 \mathrm{~min}$ before and $24 \mathrm{~h}$ after the application were made.

Standardization of measuring instruments for intraocular pressure: Goldman applanation tonometer, Haag-Streit brand. Biomicroscopy: slit lamp; any of the existing models in our Ophthalmology Department. $2.5 \mathrm{mg}(0.1 \mathrm{~mL})$ intravitreal injection of Bevacizumab was performed under sterile technique in operating room, with topical anesthetic tetracaine $3.5 \mathrm{~mm}$ from the corneoscleral limbus; the procedures were performed by medical residents at the service of Ophthalmology at Civil Hospital of Culiacán. We considered high IOP (intra ocular pressure) when exceeded $21 \mathrm{mmHg}$ or when there was a difference equal to or greater than $6 \mathrm{mmHg}$.

\section{Results and Analysis}

It was made 98 applications in a total of 67 patients, average age of 65.4 years was obtained (Table 1). Pre-application average IOP was $13.5 \pm 2.6 \mathrm{mmHg}$, and post-implementation IOP was $13.8 \pm 2.6 \mathrm{mmHg}$. There are 98 applications in a total of 67 patients, a total of 35 women, $52.2 \%$ representing sample and 32 men, $47.8 \%$ being the sample. An average age of 65.4 years was obtained with a standard deviation of 12.9 years, age range 31 89 years no statistical significance $(p=0.1810)$ was found (Fig. 1). Visual acuity was $0.763 \pm 0.600 \log$ MAR scale, before application, compared to $0.700 \pm 0.589$ average $(p=0.008)$ (Fig. 2). The most common vitreoretinal 
diseases were: PDR + MESC (proliferative diabetic retinopathy with macular edema significant clinically) at $38.8 \%$, NPDRSC (nonproliferative diabetic retinopathy significant clinical) at $20.9 \%$, macular degeneration related to age at $20.9 \%$ (Fig. 3).

Complications found were subconjunctival hemorrhage in 39 events $(39.8 \%)$, it increased IOP $>6 \mathrm{mmHg}$ in one patient.

Table 1 Patient's demographics and characteristics.

\begin{tabular}{llll}
\hline Characteristics & & Frequency $(n)$ & Percentage \\
\hline Age (mean) & & $9 \pm 12.93$ & \\
\hline \multirow{2}{*}{ Injected eye (total 98) } & OD & 48 & $48.97 \%$ \\
& OS & 50 & $51.02 \%$ \\
\hline \multirow{2}{*}{ Sex } & Female & 35 & $52.2 \%$ \\
& Male & 32 & $47.8 \%$ \\
\hline
\end{tabular}

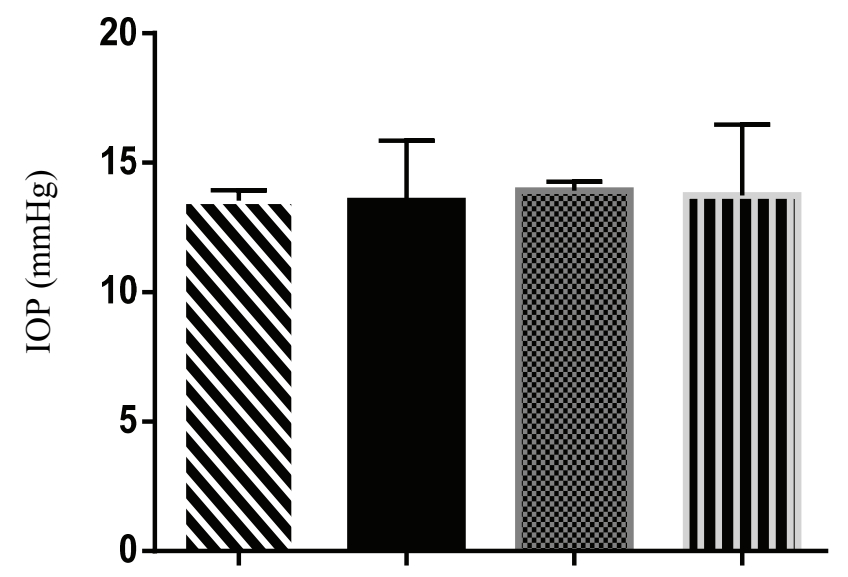

$\sim$ IOP PRE OS

- IOP PRE OD

$\triangle I O P$ POS OD

III IOP POS OS

Fig. 1 IOP (intra ocular pressure).

OD: right eye;

OS: left eye.

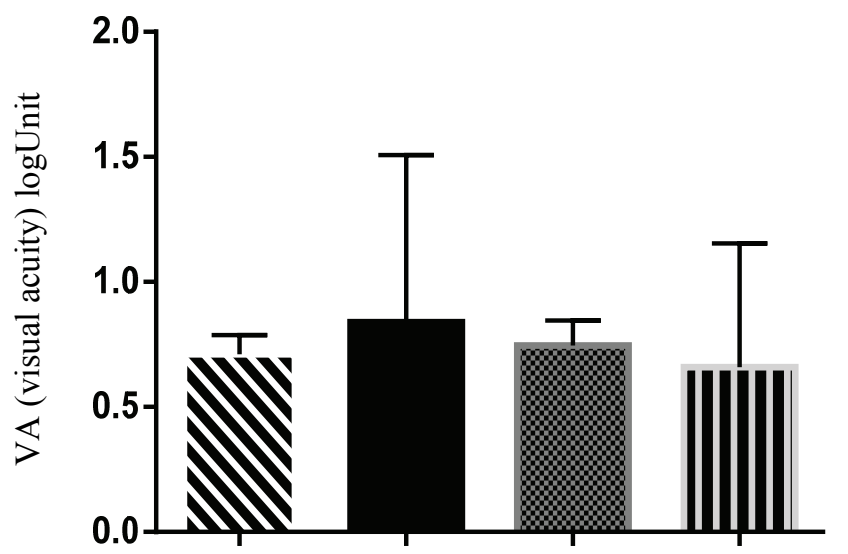

V.A. PRE OS

V.A. PRE OD

$\approx$ V.A.POS OD

II V.A.POS OS

Fig. 2 VA (visual acuity).

OD: right eye;

OS: left eye. 


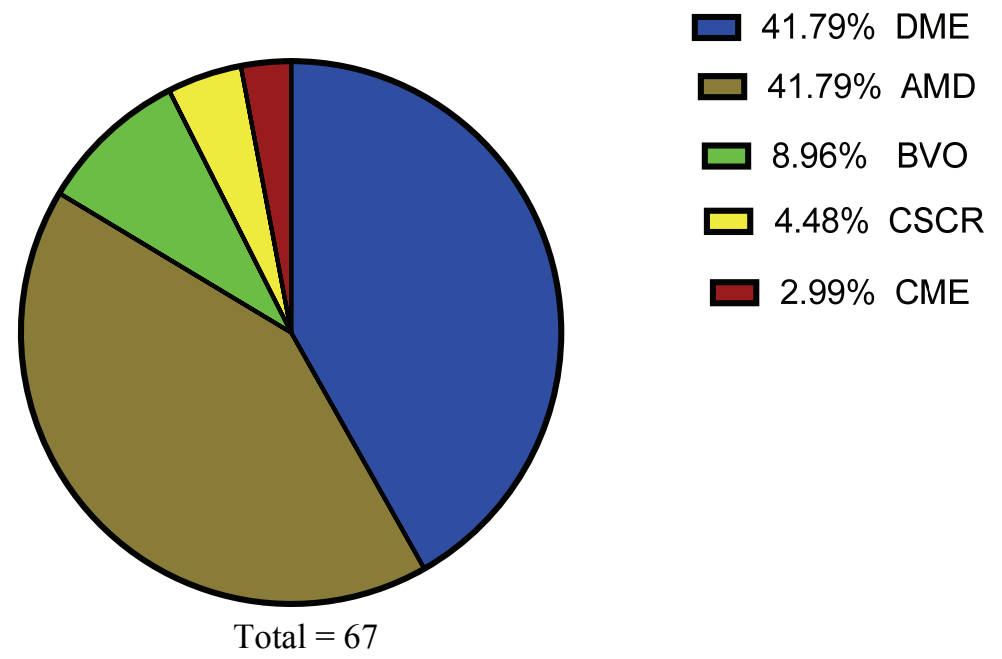

Fig. 3 Vitreoretinal diseases distribution.

DME: diabetic macular edema;

AMD: age related macular degeneration;

BVO: Branch retinal vein occlusion;

CSCR: choroidal retinopathy central serous;

CME: cystoid macular edema.

\section{Discussion}

In our study, we found no significant change in intraocular pressure before and after the application of $2.5 \mathrm{mg}(0.1 \mathrm{~mL})$ of intravitreal Bevacizumab. This is comparable to the results presented by Güler et al. [11] who analyzed the central thickness of the cornea, the simulated keratometry, the anterior chamber depth, the iridocorneal angle and intraocular pressure in patients who were applied a single dose of $2.5 \mathrm{mg}(0.1 \mathrm{~mL})$ of Bevacizumab.

\section{Conclusions}

The use of intravitreal Bevacizumab with a $2.5 \mathrm{mg} / 0.1 \mathrm{~mL}$ dose is a choice for patients with any retinal pathology with macular edema. Anti-VEGF therapy is the mainstay for the treatment of many retinal diseases. Despite its promising efficacy in halting the disease and improving the vision for the patients should consider the potential systemic and ocular risks and benefits of receiving intravitreal anti-VEGF therapy and closely monitoring the patients for adverse effects that may occur in the immediate or subsequent periods after administration of the drugs.

\section{Acknowledgments}

We would like to thank the Doctors Ophthalmology Service Residents of the HCC and CIDOCS for their cooperation for the success of this study.

\section{Conflict of Interest}

The authors report no conflicts of interest in this work.

\section{References}

[1] Folkman, J., and Klagsbrun, M. 1987. "Angiogenic Factors." Science 235: 442-7.

[2] Ferrara, N. 2009. "Vascular Endothelial Growth Factor: Basic Biology and Clinical Applications." In Current Clinical Oncology: From Local Invasion to Metastatic Cancer, edited by Leong, S. P. L. New York: Human Press, a part of Springer Science Business Media, LLC.

[3] Stahl, A. 2016. Anti-angiogenic Therapy in Ophthalmology, Essentials in Ophthalmology. Zurich: Springer.

[4] Holz, F. 2013. Age-Related Macular Degeneration. 2nd ed. Berlin: Springer.

[5] Dvorak, H. F. 2002. "Vascular Permeability Factor/Vascular Endothelial Growth Factor: A Critical 
Cytokine in Tumor Angiogenesis and a Potential Target for Diagnosis and Therapy." J. Clin. Oncol. 20 (21): 4368-80.

[6] Mintz-Hittner, H. A., Kennedy, K. A., Chuang, A. Z., et al. 2011. "Efficacy of Intravitreal Bevacizumab for Stage 3+ Retinopathy of Prematurity." N. Engl. J. Med. 364 (7): 603-15.

[7] Solomon, S. D., Lindsley, K., Vedula, S. S., Krzystolik, M. G., Hawkins, B. S. 2014. “Anti-vascular Endothelial Growth Factor for Neovascular Age-Related Macular Degeneration." Cochrane Database of Systematic Reviews 8: CD005139.

[8] Shahsuvaryan, M. L. 2015. "Pharmacovigilance in Intraocular Antiangiogenic Therapy.” International
Journal of Ophthalmic Research 1 (1): 5-10.

[9] Virgili, G., Parravano, M., Menchini, F., and Evans, J. R. 2014. "Anti-vascular Endothelial Growth Factor for Diabetic Macular Oedema." Cochrane Database of Systematic Reviews 10: CD007419.

[10] Martinez-Zapata, M. J., Martí-Carvajal, A. J., Solà, I., Pijoán, J. I., Buil-Calvo, J. A., Cordero, J. A., et al. 2014. "Anti-vascular Endothelial Growth Factor for Proliferative Diabetic Retinopathy." Cochrane Database of Systematic Reviews 11: CD008721.

[11] Güler, M., Çapkın, M., Şimşek, A., Bilak, Ş., Bilgin, B., Hakim Reyhan, A., et al. 2014. "Short-Term Effects of Intravitreal Bevacizumab on Cornea and Anterior Chamber." Current Eye Research 39 (10): 989-93. 